UTAP-264/97

\title{
Cosmological Gamma-Ray Bursts and Evolution of Galaxies
}

\author{
Tomonori Totani \\ Department of Physics, School of Science, The University of Tokyo, Tokyo 113, Japan \\ E-mail: totani@utaphp2.phys.s.u-tokyo.ac.jp \\ To Appear in ApJ Letters \\ (Received 1997 May 15; Accepted 1997 July 2)
}

\begin{abstract}
Evolution of the rate density of cosmological gamma-ray bursts (GRBs) is calculated and compared to the BATSE brightness distribution in the context of binary neutron-star mergers as the source of GRBs, taking account of the realistic star formation history in the universe and evolution of compact binary systems. We tried two models of the evolution of cosmic star formation rate (SFR): one is based on recent observations of SFRs at high redshifts, while the other is based on a galaxy evolution model of stellar population synthesis that reproduces the present-day colors of galaxies. It is shown that the binary merger scenario of GRBs naturally results in the comoving rate-density evolution of $\propto(1+z)^{2-2.5}$ up to $z \sim 1$, that has been suggested independently from the compatibility between the number-brightness distribution and durationbrightness correlation. If the cosmic SFR has its peak at $z \sim 1-2$ as suggested by recent observations, the effective power-index of GRB photon spectrum, $\alpha \gtrsim 1.5$ is favored, that is softer than the recent observational determination of $\alpha=1.1 \pm 0.3$. However, high redshift starbursts $(z \gtrsim 5)$ in elliptical galaxies, that have not yet been detected, can alleviate this discrepancy. The redshift of GRB970508 is likely about 2, just below the upper limit that is recently determined, and the absorption system at $z=0.835$ seems not to be the site of the GRB.
\end{abstract}

Subject headings: binaries: close - stars: neutron-cosmology: observationsgalaxies: evolution - gamma rays: bursts 


\section{Introduction}

It is well known that spatial distribution of the classical gamma-ray bursts (GRBs) detected by the Burst and Transient Source Experiment (BATSE; Meegan et al. 1996) is isotropic with high precision but the number of weak bursts is significantly deficient compared with the Euclidean distribution (Meegan et al. 1992), suggesting that GRBs are located at cosmological distances. Furthermore, recent discovery of an optical transient source embedded in an extended object for GRB970228 (e.g., van Paradijs et al. 1997; Sahu et al. 1997), or metal absorption lines at $z=0.835$ for GRB970508 (Metzger et al. 1997) finally confirmed the cosmological origin of GRBs. The observed $\log N-\log P$ distribution, where $N$ is the observed number of GRBs with peak photon flux larger than $P\left[\mathrm{~cm}^{-2} \mathrm{~s}^{-1}\right]$, agrees well with a cosmological distribution (Mao \& Paczyński 1992; Piran 1992; Dermer 1992 ) if the faintest bursts are located at redshift of $z \sim 1$ and the comoving GRB rate density is constant with time. The effect of possible GRB rate evolution has also been discussed in a number of papers (see, e.g., Cohen \& Piran 1995; Rutledge, Hui, \& Lewin 1995) using some analytic forms of GRB rate evolution, e.g., $R_{\mathrm{GRB}} \propto(1+z)^{\beta}$.

The best candidate for the cosmological origin of GRBs is widely considered to be mergers of binary neutron stars (see, e.g., Narayan, Paczyński, and Piran 1992, and references therein). Lipunov et al. (1995) analyzed the BATSE $\log N-\log P$ distribution based on this scenario, taking account of the evolution of binary merger rate calculated by detailed Monte-Carlo simulations of the binary system evolution. On the other hand, the cosmic evolution of star formation rate (SFR) also plays an important role in predicting the cosmic evolution of compact binary merger rate. Lipunov et al. classified all galaxies into elliptical and spiral galaxies, and assumed initial star bursts for ellipticals and constant SFR for spirals. Recent progress of observation of high redshift galaxies, however, gives more detailed information on the cosmic star formation history (Lilly et al. 1996; Madau et al. 1996). The Canada-France Redshift Survey (CFRS) revealed a marked evolution of $2800 \AA$ luminosity density, that is considered to be a star formation indicator, as $\mathcal{L}_{2800} \propto(1+z)^{3.9 \pm 0.75}$ to $z \sim 1$ (for $\Omega_{0}=1$, Lilly et al. 1996). The constant SFR approximation in spiral galaxies is therefore no longer justified even at $z<1$.

Because the star formation history in a galaxy is strongly correlated to its present-day colors or spectra, that are different among the morphological types of galaxies, it is also possible to construct a model of cosmic star formation history based on the spectrum of local galaxies by using galaxy evolution models of stellar population synthesis (Totani, Sato, \& Yoshii 1996; Totani, Yoshii, \& Sato 1997). In this Letter, we analyze the BATSE $\log N-\log P$ distribution based on the realistic models of the cosmic star formation history

in the context of the compact binary mergers as the source of GRBs, taking account of the 
evolution of compact binaries due to the gravitational wave radiation. We use two models of the star formation history: one is based on the recent observations of high redshift SFRs, and the other is a theoretical model based on the galaxy evolution models of stellar population synthesis (Arimoto \& Yoshii 1987; Arimoto, Yoshii, \& Takahara 1992, hereafter AYT).

\section{Models of Cosmic GRB Rate History}

The cosmic evolution of comoving merger-rate density of binary neutron stars $\left(R_{G R B}\right)$ as a function of cosmic time $t_{c}$ is given by a convolution of cosmic SFR density $\left(R_{*}\right)$ and probability distribution of the time from formation to merger of a binary system $\left(P_{m}\right)$, i.e.,

$$
R_{\mathrm{GRB}}\left(t_{c}\right) \propto \int_{t_{F}}^{t_{c}} d t^{\prime} R_{*}\left(t^{\prime}\right) P_{m}\left(t_{c}-t^{\prime}\right)
$$

where $t_{F}$ is the formation epoch of galaxies. We have assumed here the binary formation rate is proportional to the star formation rate. Because the massive stars in high-mass binary systems evolve into double neutron stars with much shorter time scale than the typical time scale of galaxy evolution or the Hubble time $(1-10$ Gyrs $)$, the form of $P_{m}$ is essentially determined by the initial distribution of the separation between two neutron stars. Since a compact binary with separation $a$ will merge in a time $t \propto a^{4}$ by gravitational wave radiation, $P_{m}(t)$ can be written as

$$
P_{m}(t) \propto \frac{d n}{d t}=\frac{d n}{d a} \frac{d a}{d t} \propto t^{\gamma / 4} t^{-3 / 4}
$$

where we assumed the initial separation distribution as $d n / d a \propto a^{\gamma}$. If we assume $\gamma=-1$, as observed in the distribution of initial separation of normal binaries (i.e., double main-sequence stars; Abt 1983), $P_{m}$ is proportional to $t^{-1}$. Although there is few observational information on $d n / d a$ of compact binaries, the dependence of $P_{m}(t)$ on the uncertain $\gamma$ is small because of the fourth-power dependence of merger time on $a$. There

are some calculations of $P_{m}$ in a more sophisticated way using population synthesis models of stellar binary systems (Tutukov \& Yungelson 1994; Lipunov et al. 1995), and their calculations also show that $P_{m}$ is approximately described by the form $P_{m} \propto t^{-1}$, with the lower cut-off of $t_{l} \sim 0.02$ Gyr that corresponds to initial separation of $\sim 1 R_{\odot}$. In the following analysis, we assume that $P_{m}(t) \propto t^{-1}$ when $t \geq t_{l}=0.02 \mathrm{Gyr}$, while $P_{m}=0$ when $0 \leq t<t_{l}$.

In the upper panels of Fig. 1, we show the models of cosmic star formation history used in this Letter. Fig. 巴 (a) shows the "observational" model of comoving SFR density 
evolution, with recent observational estimates of the SFR density at high redshifts (Madau et al. 1996). In the range of $z=0-1$, the model is adjusted to the data of the CFRS (Lilly et al. 1996), and the model SFR beyond $z=1$ is not just a simple extrapolation, but it is based on the evolution of neutral hydrogen gas in damped Ly $\alpha$ systems seen in quasar spectra that implies a peak of cosmic SFR around $z \sim 1-2$ (Pei \& Fall 1995). The observed SFR density, that is inferred from the luminosity density of galaxies, generally depends on the cosmological parameters $\left(\Omega_{0}\right.$ and $\left.\lambda_{0}\right)$, and we assume the same dependence of the SFR evolution on $\Omega_{0}$ and $\lambda_{0}$ as that of luminosity density. Fig. 目 (b) shows the comoving rate-density of GRBs calculated by Eq. (1) with the SFR models shown in Fig. 1 (a), for which we normalize the present GRB rate to the unity. (The absolute values of GRB rates are determined by the fit to the BATSE data.) An important result is that the GRB rate evolves rapidly from $z=0$ to $z \sim 1$, and has its peak at $z \sim 1-2$. If we parametrize the evolution of GRB rate as $(1+z)^{\beta}$, these calculations imply that $\beta$ lies in a range of $2-2.5$ at $z \lesssim 1$, depending on the cosmological parameters.

Fig. 1 (c) shows the SFR evolution calculated from the population synthesis model of galaxy evolution. For details of the calculation, see Totani et al. (1996; 1997). The SFR evolution is given as a function of time from the galaxy formation, and four different curves correspond to the four variations of the galaxy evolution model for spiral galaxies: S1, S2, I1, and I2. The symbol 'S' refers to the simple, closed-box model, while 'I' to the infall model that allows for material infall into the disk region. The number attached to 'S' and ' $\mathrm{I}$ ' is the adopted value of power index in the Schmidt law of star formation (for details see AYT). These four models are used to assess the uncertainties in the star formation history of the galaxy evolution model. The model for elliptical galaxies is a so-called galactic wind model, in which stars are formed by the initial star bursts and there is no star formation after the galactic wind that occurs about 1 Gyr after the galaxy formation. The SFR in the universe is dominated by ellipticals during the first 1 Gyr after the formation. The GRB rate evolutions calculated from these models of SFR evolution are shown in Fig. 1 (d).

The observational SFR evolution [Fig. [1 (a)] and that of the galaxy evolution model [Fig. 11 (c)] are consistent with each other at $z \lesssim 1$ where SFR is dominated by spiral galaxies, provided that there is non-vanishing cosmological constant, $\lambda_{0}$ (Totani, Yoshii, \& Sato 1997). However, beyond $z \sim 2-3$ or during $\sim 1$ Gyr after the galaxy formation, these two models give completely different behaviors because the initial star bursts of giant elliptical galaxies are included in the galaxy evolution model while the signature of such bursts has not yet been detected. Some interpretations for this problem are proposed, but here we take the picture that giant elliptical galaxies formed at $z \gtrsim 5$ and no star formation occurs at observed redshifts (Totani, Yoshii, \& Sato 1997; Maoz 1997). We will discuss later the possibility that the GRB peak flux distribution can provide some information on 
the formation epoch of elliptical galaxies.

\section{Results: Comparison of GRB Rate Evolution and the BATSE data}

For the comparison of the predicted GRB rate evolution and the BATSE data, we use the number versus peak flux distribution of the BATSE 3B catalog (Meegan et al. 1996) measured in the energy range of $50-300 \mathrm{keV}$ by $1024 \mathrm{msec}$ time scale. We set the analysis threshold of $P_{t h}=0.4$ [photons $\mathrm{cm}^{-2} \mathrm{~s}^{-1}$ ] above which the detection efficiency is almost $100 \%$, and there are 665 GRBs above this threshold. We assume neither dispersion nor evolution of the intrinsic luminosity of GRBs (standard candle approximation). The detection rate of GRBs $(N)$ whose peak flux is larger than $P$ is calculated as follows:

$$
N(>P)=\int_{0}^{z(P)} \frac{d V}{d z} \frac{R_{\mathrm{GRB}}(z)}{(1+z)} d z
$$

where $d V / d z$ is the comoving volume element per unit $z$, and $z(P)$ is a redshift that corresponds to the peak flux $P$. The factor of $1 /(1+z)$ is included to account for the time dilation of the interval between detected bursts. The value of $z(P)$ is given as the solution of the following equation,

$$
\frac{P}{P_{t h}}=\left(\frac{1+z}{1+z_{t h}}\right)^{2-\alpha}\left(\frac{d_{L}\left(z_{t h}\right)}{d_{L}(z)}\right)^{2},
$$

where $d_{L}$ is the luminosity distance, $z_{t h}$ the redshift corresponding to $P_{t h}$ (hereafter $z_{0.4}$ ), and $\alpha$ the effective power-index of photon number spectrum of GRBs $\left(d N / d E_{\gamma} \propto E_{\gamma}^{-\alpha}\right)$. A recent investigation by Mallozzi, Pendleton, \& Paciesas (1996, hereafter MPP96) showed that the range of $\alpha$ appropriate for the $\log N-\log P$ analysis is $1.1 \pm 0.3$. However, the dispersion of spectral index is considerably large within individual bursts, and we will try a wide range of $\alpha$ in the following, considering the MPP96 range to be feasible.

We use the Kolmogorov-Smirnov test for comparison between the models and the BATSE $\log N-\log P$ distribution. Fig. 2 shows the allowed regions obtained by this test as contour maps of $z_{0.4}$ and $\alpha$, for the three representative cosmological models. The "observational" GRB rate model is used in the left panels, while the GRB rate calculated from the galaxy evolution model (I1) in the right panels. The formation redshift of galaxies is assumed to be $z_{F}=5$ for all types of galaxies and the effect of changing $z_{F}$ is small as long as the redshift of the faintest bursts observed by BATSE is less than $\sim 3$. We have chosen $H_{0}$ so as to set the age of galaxies to a reasonable value (12 Gyrs). A general trend is that the allowed range for $z_{0.4}$ becomes smaller with increasing $\alpha$, because larger values of $\alpha$ (i.e., softer spectra) make the cosmological effect stronger, and $z_{0.4}$ has to become 
smaller to compensate the cosmological effect. The left panels of this figure show that the observational SFR history favors $\alpha \gtrsim 1.5$ because of the rapid decline of $R_{G R B}(z)$ beyond $z \sim 2$, and this is inconsistent with the determination by MPP96, although the significance of this discrepancy is difficult to assess due to the uncertainties in estimation of $\alpha$ as well as in SFR observations. On the other hand, the results of the GRB rate derived from the galaxy evolution model (right panels) show that the existence of high redshift starbursts in elliptical galaxies alleviates the above discrepancy, because the GRB rate continues to increase toward the past to redshift larger than $\sim 2-3$. There are allowed regions for $z_{0.4} \sim$ 2-3 with the spectral index determined by MPP96. The spiral models other than I1 give qualitatively similar results, and $z_{0.4}$ varies by about $\lesssim 1$ with changing models.

In Fig. 3 we show some examples of the predicted $\log N-\log P$ distribution (upper panel) and corresponding $z-P$ relation (lower panel) for the two SFR-evolution models: the galaxy evolution model (thin-solid, short- and long-dashed lines) and the observational SFR model (dot-dashed line). The adopted values of $\Omega_{0}, \lambda_{0}, z_{0.4}$, and $\alpha$ are shown in the figure. All the four curves are consistent with the BATSE data (see Fig. 2), and have the roughly Euclidean slope $(-3 / 2)$ at $P \gtrsim 10 \mathrm{~cm}^{-2} \mathrm{~s}^{-1}$ that is consistent with the Pioneer Venus Orbiter data (Fenimore et al. 1993). If it is the case that elliptical galaxies formed before $z \sim 5$, the observed number of GRBs will continue to increase with decreasing flux below the BATSE detection limit. On the other hand, if the cosmic SFR is actually peaked at $z \sim 1-2$, we will see rapid decline of the number of GRBs below the BATSE limit (dot-dashed line). In any case, the redshift corresponding to a peak flux increases rapidly with decreasing flux below the BATSE limit, and future experiments with greater sensitivity will provide valuable information on the formation epoch of galaxies, especially for ellipticals.

\section{Discussion \& Conclusions}

Recent discovery of the metal absorption lines in the optical counterpart associated with GRB970508 provides us an important and independent constraint on the distance to the source of the GRB: $0.835 \leq z<2.1$ (Metzger et al. 1997). We calculate the peak fluxes in the BATSE range (50-300 keV) for GRB970508 (Kouveliotou et al. 1997), as well as for GRB970228 (Costa et al. 1997; see also Piro et al. 1997 for the conversion of BeppoSax count rate into photon flux), assuming $\alpha=1$.1. The fluxes are indicated in Fig. 3. A striking implication of our calculation is that the redshift of GRB970508 is likely $\sim 2$, just

below the upper limit of Metzger et al. Although the possible dispersion in GRB luminosity function may allow lower redshifts, it seems difficult to consider the absorption system at 
$z=0.835$ as the GRB source, as long as we assume that GRBs are produced by binary neutron-star mergers.

Some cosmological GRB models other than the compact binary mergers, e.g., failed Ib supernovae (Woosley 1993), predict the GRB rate evolution that is proportional to SFR. We analyzed the BATSE data with the GRB rate proportional to the models of the cosmic SFR history used in this Letter. The GRB rate evolution at $z=0-1$ becomes steeper than the case of binary mergers because of the lack of time lag during the spiral-in of compact binaries by gravitational wave radiation, and this requires larger $z_{0.4}$ or $\alpha$ to compensate the steeper evolution of $R_{G R B}$. Therefore the allowed values for $\alpha$ become even softer, apart from the determination of MPP96. Especially, with the observational SFR evolution, we found no acceptable fit with $\alpha<1.5$ with $95 \%$ C.L., and it can be concluded that the compact binary scenario is more favorable than GRB models that predict a GRB rate proportional to SFR.

We have not considered the normalization of $R_{G R B}$ from the SFR models, because the normalization does not affect the $\log N-\log P$ analysis. However the comparison of the model $R_{G R B}$ and observed number of GRBs gives an important consistency check. The fit of our typical $R_{G R B}$ models to the BATSE data implies that the GRB rate at $z=0$ is $\sim 7 \times 10^{-9} h^{3}$ $\left[\mathrm{yr}^{-1} \mathrm{Mpc}^{-3}\right]$ where $h=H_{0} /(100 \mathrm{~km} / \mathrm{s} / \mathrm{Mpc})$. On the other hand, assuming the Salpeter IMF, flat distribution of the mass ratio of binary systems, and binary formation rate equal to SFR, we can estimate the binary merger rate expected from the cosmic SFR history, that becomes about $6 \times 10^{-6} h^{2}\left[\mathrm{yr}^{-1} \mathrm{Mpc}^{-3}\right]$ at $z=0$. The expected rate is therefore $\sim 10^{3}$ times larger than the observation of BATSE, and the beaming of GRBs with $d \Omega \sim 4 \pi 10^{-3}$ may be required. This result is consistent with the previous estimate of Lipunov et al. (1995), although it should be noted that the above estimate has large uncertainties in SFR itself or conversion of SFR into merger rate.

We have shown in this Letter, for the first time, that the binary neutron-star merger scenario of the cosmological GRBs naturally results in the comoving rate-density evolution of GRBs roughly proportional to $(1+z)^{2-2.5}$ at low redshifts $(z \lesssim 1)$. It should be noted that such evolution has been suggested independently from the compatibility between the $\log N-\log P$ analysis and time dilation analysis (Horack, Emslie, \& Hartmann 1995; Horack, Mallozzi, \& Koshut 1996; Mészáros \& Mészáros 1996). In fact, the time dilation factor, $\left(1+z_{\text {dim }}\right) /\left(1+z_{\text {bright }}\right)$, for the bright and dim+dimmest bursts defined by Norris et al.

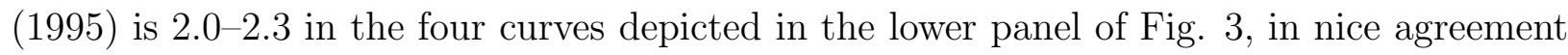
with the result of Norris et al. (1995).

The author is grateful to an anonymous referee for valuable comments. He also thanks A. Mészáros for useful comments. This work has been supported in part by the 
Grant-in-Aid for the Scientific Research Fund (3730) of the Ministry of Education, Science, and Culture in Japan.

\section{REFERENCES}

Abt, H., 1983, ARA\&A, 21, 343

Arimoto, N. \& Yoshii, Y. 1987, A\&A, 173, 23

Arimoto, N., Yoshii, Y., \& Takahara, F. 1992, A\&A, 253, 21 (AYT)

Cohen, E. \& Piran, T. 1995, ApJ, 444, L25

Costa, E. et al. 1997, IAU Circ. 6572

Dermer, C. D. 1992, Phys. Rev. Lett., 68, 1799

Fenimore, E. E. et al. 1993, Nature, 366, 40

Horack, J. M., Emslie, A. G., \& Hartmann, D. H. 1995, ApJ, 447, 474

Horack, J. M., Mallozzi, R. S., \& Koshut, T. M. 1996, ApJ, 466, 21

Kouveliotou, C. et al. 1997, IAU Circ. 6660

Lilly, S. J., Fèvre, O. Le., Hammer, F., \& Crampton, D. 1996, ApJ, 460, L1

Lipunov, V. M. et al. 1995, ApJ, 454, 593

Madau, P. et al. 1996, MNRAS, 283, 1388

Mallozzi, R. S., Pendleton, G. N., \& Paciesas, W. S. 1996, ApJ, 471, 636 (MPP96)

Mao, S. \& Paczyński, B. 1992, ApJ, 388, L45

Maoz, D. 1997, astro-ph/9704173

Meegan, C. A. et al. 1992, Nature, 355, 143

Meegan, C. A. et al. 1996, ApJS, 106, 65

Mészáros, A. \& Mészáros, P. 1996, ApJ, 466, 29

Metzger, M. R. et al. 1997, IAU Circ. 6655 
Narayan, R., Paczyński, B., \& Piran, T. 1992, ApJ, 395, L83

Norris, J. P. et al. 1995, ApJ, 439, 542

Pei, Y. C. \& Fall, S. M. 1995, ApJ, 454, 69

Piran, T. 1992, ApJ, 389, L45

Piro, L. et al. 1997, IAU Circ. 6467

Rutledge, R. E., Hui, L. \& Lewin, W. H. G. 1995, MNRAS, 276, 753

Sahu, K.C. et al. 1997, Nature, 387, 476

Totani, T., Sato, K. \& Yoshii, Y. 1996, ApJ, 460, 303

Totani, T., Yoshii, Y., \& Sato, K. 1997, ApJ, 483, L75

Tutukov, A. V. \& Yungelson, L. R. 1994, MNRAS, 268, 871

van Paradijs, J. et al. 1997, Nature, 386, 686

Woosley, S. E. 1993, ApJ, 405, 273

This preprint was prepared with the AAS IATEX macros v4.0. 


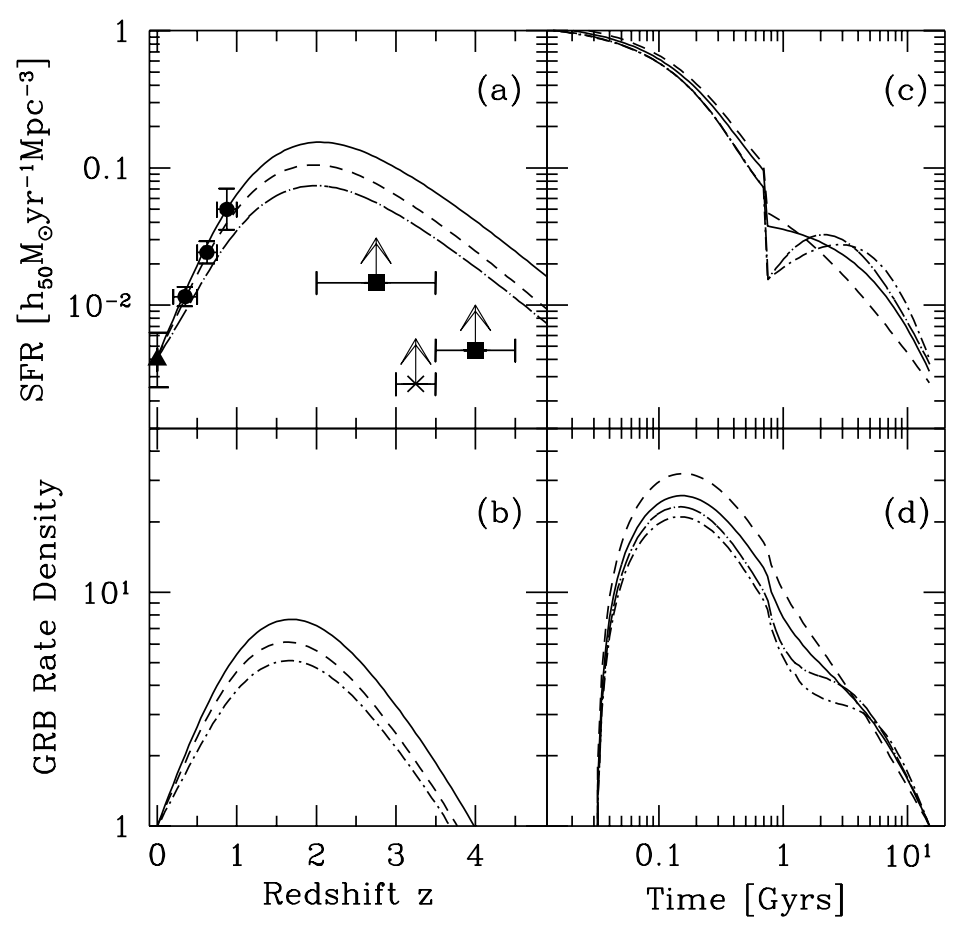

Fig. 1.- (a) Data points are the recent measurements of comoving density of star formation rate (SFR) (Madau et al. 1996, for $\Omega_{0}=1$ universe), and the curves are the models of SFR history based on these observations (see text). The cosmological parameters of $\left(\Omega_{0}, \lambda_{0}\right)=(1,0),(0.2,0)$ and $(0.2,0.8)$ are used for the solid, dashed, and dot-dashed lines, respectively. (b) Models of the comoving rate-density evolution of GRBS calculated from the SFR history shown in $(a)$. The curves are normalized at $z=0$. (c) SFR history calculated from the galaxy evolution model of population synthesis as a function of time from galaxy formation. Four curves represent the models of spiral galaxies: S1 (solid), S2 (dashed), I1 (dot-short-dashed) and I2 (dot-long-dashed) (see text for detail). (d) GRB rate evolution calculated from the SFR history shown in $(c)$. The curves are normalized at 12 Gyr. 


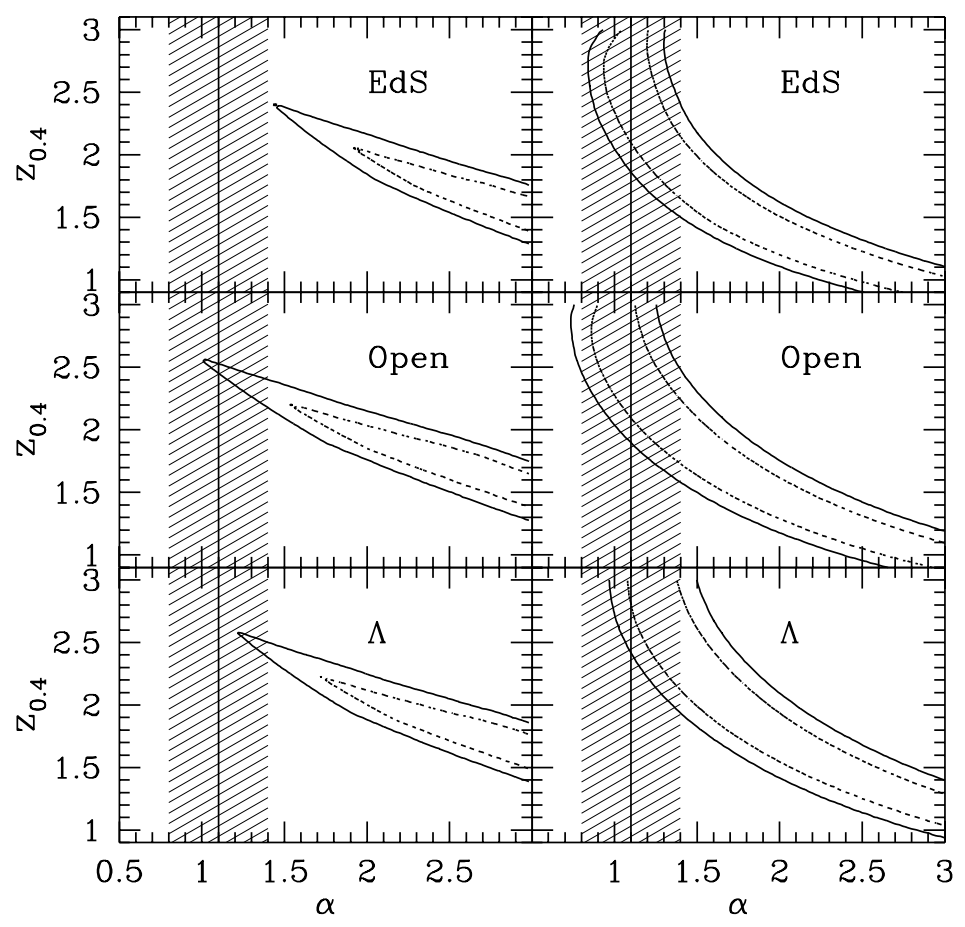

Fig. 2.- Allowed regions for the effective power-index of GRB spectra $(\alpha)$ and the redshift corresponding to the BATSE peak flux of $0.4\left[\mathrm{~cm}^{-2} \mathrm{~s}^{-1}\right]\left(z_{0.4}\right)$ obtained from the $\log N-\log P$ analysis of the BATSE data. The dotted lines are for 68 \% C.L. regions, while the solid lines for $95 \%$ C.L. The observational SFR-evolution model is used in the left panels, while the SFR of galaxy evolution model (I1) is used for right panels. The cosmological parameters of $\left(\Omega_{0}, \lambda_{0}\right)=(1,0),(0.2,0)$ and $(0.2,0.8)$ are used for the top, middle, and bottom panels, respectively. The shaded regions are the feasible range of $\alpha$ determined by the BATSE data (Mallozzi, Pendleton, \& Paciesas 1996). 


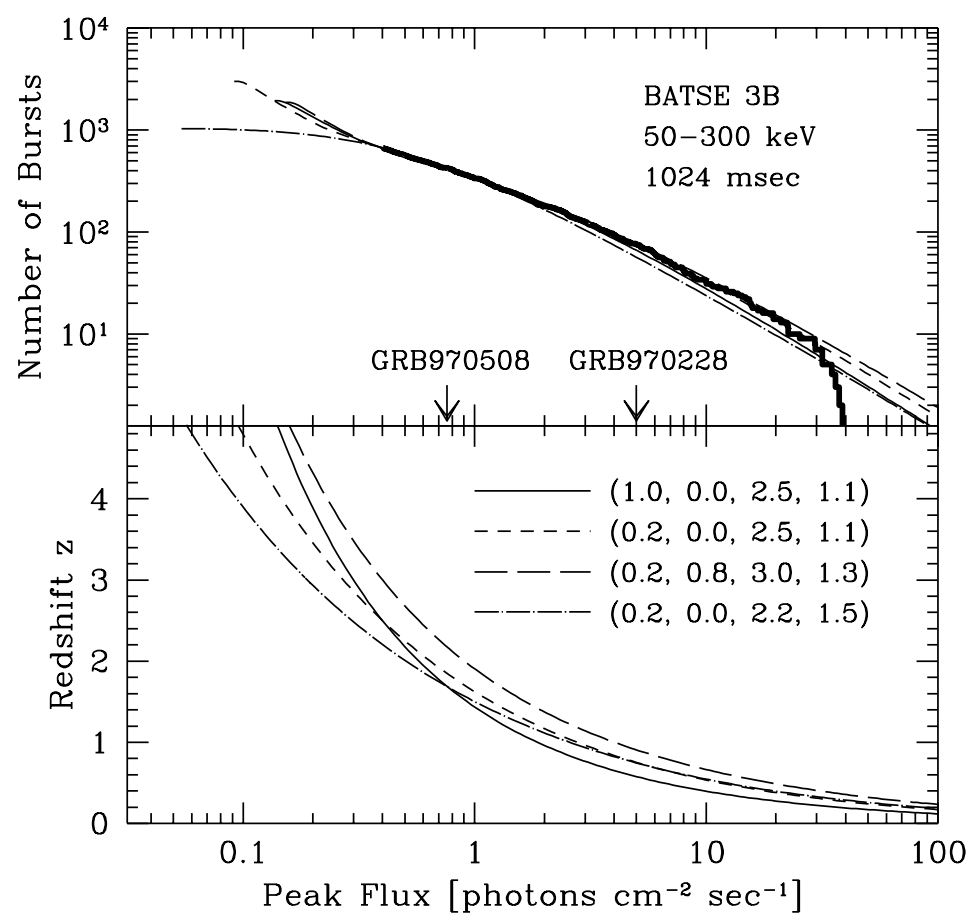

Fig. 3.- The number versus peak-flux distribution (upper panel) and redshift versus peakflux relation (lower panel). Thick solid line is the observation by BATSE (Meegan et al. 1996). Theoretical curves are calculated with SFR of the galaxy evolution model (thin-solid, long- and short-dashed lines) and with the observational SFR-evolution model (dot-dashed line). The line markings are the same for upper and lower panels. The adopted values of $\Omega_{0}, \lambda_{0}, z_{0.4}$, and $\alpha$ are shown in the figure. The peak fluxes of GRB970228 and GRB970508 are also indicated. 